\title{
Martin Löw-Beer
}

\section{Ist „Zwanghaftes Handel“ ein paradoxer Begriff? Ein Versuch der Beschreibung von Zwangssymptomen}

\begin{abstract}
What does it mean that a person is psychologically forced to do certain things? It is argued that there are, strictly speaking, no compulsive actions. Talking of compulsive actions people refer to intentional actions that are means of avoiding irrational panic attacks. People know that certain situations will cause them irrational fears and that is the reason why they avoid these situations. These irrational fears are either mediated by wrong perceptions or by emotional delusions. In the former case the people believe wrongly that they are in danger, while in the latter case they know that they "feel wrong", but cannot help it. The compulsive elements cannot be found in the actions but in the irrational fears and the obsessions that are part of them.
\end{abstract}

Wenn wir zu der Überzeugung kommen, daB jemand aus innerem Zwang handelt, so ändert sich unsere Einstellung ihm gegenüber. Strawson (1978, 201) hat bemerkt, da $B$ wir dazu tendieren, ihm unter diesen Umständen Handlungen, die unseren Erwartungen nicht entsprechen, nicht ubelzunehmen. Das ist deshalb so, weil man der Uberzeugung ist, daB der Zwanghafte nicht anders handeln konnte und deswegen nicht verantwortlich zu machen ist. Strawson meint, daß der Verweis auf Zwanghaftigkeit zwar einerseits exkulpiert, aber andererseits Gleichstellung und Achtung einer Person gefährdet. Die Meinung, da $B$ jemand zwanghaft ist, führt partiell dazu, ihn als Person zu entwerten. Wir stellen uns berechnend, kontrollierend, möglicherweise auch behandelnd auf seine Reaktionen ein, nehmen ihn aber nicht mehr als autonome Person ernst. Wir neigen dazu, uns nicht mehr emotional und intellektuell mit ihm auseinanderzusetzen, betrachten seine Reaktionen stattdessen als Probleme für Kontrolle und Erklärungen. So nimmt es nicht Wunder, daB Ansätze, die versuchen, Kriterien für psychische Störungen zu nennen, u.a. vom Begriff "Zwanghaftigkeit" Gebrauch machen.

\section{Zwanghaftigkeit und psychische Krankheit}

Im wesentlichen gibt es drei Vorschläge, psychische Krankheit zu begreifen: 
I) Psychische Krankheit impliziert eine Störung der Fähigkeit, sich an soziale oder natürliche Umwelten anzupassen. Anpassung kann man entweder aus der Perspektive sozialer Systeme begreifen oder aus der Perspektive von Individuen, sei es biologisch unter den Imperativen von Selbsterhaltung und Reproduktion, sei es psychologisch, indem man die Entwicklung der Fähigkeit untersucht, Bedürfnisse, die jeder hat, zu befriedigen, z.B. Bedürfnisse nach Lust, Wohlergehen und Sicherheit.

2) Psychische Krankheit ist ein psychisches Leid, das Menschen ohne gute Gründe ertragen müssen und das sich auf der Basis mentaler Ursachen erhält.

3) Psychische Krankheit ist eine psychisch verursachte Beeinträchtigung von Freiheit. Mentale Krankheit hat dann etwas mit der Behinderung der Entfaltung von Tätigkeiten und der Einengung des Handlungsspielraums von Personen zu tun.

Ich will diese Vorschläge weder begründen noch kritisieren, sondern nur darauf hinweisen, daß der Phänomenbereich, den ich thematisieren möchte, nämlich Zwanghaftigkeit, ein Element aller dieser Klassen von Krankheitsdefinitionen ist.

Denn ad I) schränkt Zwanghaftigkeit Flexibilität und Lernfähigkeit ein und beschneidet somit die Fähigkeit zur Anpassung und Selbsterhaltung. Ad 2) schränkt Zwanghaftigkeit die Fähigkeit von Personen ein, zu tun was sie wollen, und dies impliziert Leid. Ad 3 ) ist schlieBlich selbstverständlich, daß Zwang Freiheit einschränkt, wenn auch unklar ist, was unter 'innerer Freiheit' zu verstehen ist.

\section{Zwanghaftigkeit und Handeln}

Das Feld von Beispielen für das, was in der Psychologie 'Zwanghaftigkeit' genannt wird, ist weit. Es reicht von bizarren Handlungen wie Waschzwang über Suchthandlungen, in denen Personen sich zu Verhaltensweisen innerlich gezwungen fühlen, von denen sie wissen, daß sie ihnen schaden, Phobien, durch die auf der Basis von irrationalen Ängsten der Handlungsspielraum eingeengt wird, bis zu Handlungen, die auf der Basis von unbewuBtem Zwang erfolgen. Im letzteren Fall sind die klarsten Beispiele diejenigen, in denen Personen unter dem Einfluß von Hypnose unbewuBt Befehlen folgen. Als 'zwanghaft' werden nicht blo $B$ Handlungszusammenhänge klassifiziert, sondern auch Personen, nämlich dann, wenn Zwanghaftigkeit ein nicht konflikthaft erlebter wesentlicher Charakterzug ist. Beispiele sind Personen, die auf Ziele so festgelegt sind als ob sie Befehle wären, deren Nichtbefolgung schwere Strafen nach sich zieht. Auch Paranoiker sind in gewisser Hinsicht zwanghaft: $\mathrm{da} B$ eine feindselige Umgebung sich gegen sie 
verschworen hat, ist der unerschütterliche Hintergrund für ihr Leben. In solchen Fällen kann man stereotype, unangemessene Interpretationen von Situationen als zwanghaft bezeichnen.

Mein Thema im folgenden ist ausschlieBlich bewuBte, konflikthaft erlebte Zwanghaftigkeit. Und hier auch wiederum nur die Beschreibung, nicht die Erklärung dieser Phänomene. Ob Zwangssymptome möglicherweise unbewuBte Ursachen haben, muB bei einer solchen Untersuchung offen bleiben. Jedoch ist eine Beschreibung von Zwangssymptomen für die psychologische Theoriebi dung nicht unwichtig. Denn Voraussetzung für sie ist ja die Klarheit über den Erklärungsgegenstand. Worin liegt die Schwierigkeit bei einer Beschreibung der Zwangssymptomatik?

Es liegt nahe, zwanghaftes Handeln als Verhalten zu definieren, von dem gilt, "da $B$ die Person so handeln muB, ob sie will oder nicht" (Tugendhat 1984, 54). Derselbe Autor grenzt in einem anderen Zusammenhang Handlungen von bloßen Geschehnissen jedoch durch die folgende Definition ab: "Wo etwas getan, gehandelt wird, haben wir es mit einem Proze B zu tun, bei dem, ob die nächste Phase des Prozesses durchlaufen wird oder nicht, davon abhängt, ob der Handelnde es will." (Tugendhat 1979, 211) Der Widerspruch zwischen diesen Definitionen geht auf keine Idiosynkrasie dieses Autors zurück, sondern sie macht auf eine Schwierigkeit mit dem Begriff zwanghaften Handelns aufmerksam. Denn es entspricht dem weithin akzeptierten Handlungsbegriff, da B Handeln gewollt ist und einen Entscheidungsspielraum impliziert. Entscheidungen können nicht nur vor dem Handeln getroffen, sondern auch währenddessen revidiert werden. Sowohl der Begriff eines nichtgewollten Handelns als auch der eines Handelns ohne Alternative, und sei es bloB die Alternative nicht zu handeln, scheinen in sich widersprüchlich zu sein. Wie aber, wenn nicht als ungewolltes, subjektiv alternativeloses Handeln, läßt sich zwanghaftes Verhalten beschreiben?

Thesenhaft könnte man an die folgenden Alternativen denken:

I) Zwanghaftigkeit ist kein Handeln, sondern ein reflexartiges Verhalten, so etwas wie ein Tick. Zwanghaftigkeit käme einem Kontrollverlust gleich, der z.B. darin besteht, daB sich die Wünsche einer Person Ausdruck verschaffen, ohne daB die Person dies intendiert.

2) Zwanghaftigkeit ist ein unfreiwilliges Handeln. Nur freiwilliges Handeln schlieBt die Fähigkeit zur Wahl zwischen Alternativen ein. Verhalten im Kontext von Süchten und Phobien sind paradigmatische Beispiele für ein Handeln, das Akteure zu vollziehen haben, ob sie wollen oder nicht. Aber auch wenn sich der Begriff eines Handelns ohne Alternative als unsinnig herausstellen sollte, läßt sich möglicherweise graduell ein Verlust von Wahlmöglichkeiten konstatieren. Danach könnte der Zwanghafte zwar 
zwischen Alternativen wählen, aber er könnte nicht jene Alternative ergreifen, die ihm als die Beste erscheint. Nach dieser These lieBe sich Zwanghaftigkeit durch einen graduellen Verlust an Kontrollfähigkeit erläutern.

3) Zwanghaftigkeit hat weniger mit dem Verlust der Fähigkeit zu wählen als mit dem, was zur Wahl steht, zu tun. Die Betroffenen sind gezwungen, sich mit irrationalen Alternativen auseinanderzusetzen. Der Zwanghafte leidet demnach nicht an einem Verlust von Kontrollfähigkeit, sondern an irrationalen Problemen. Nicht sein Handeln ist zwanghaft, sondern psychische Elemente, die sein Handeln motivieren.

Zwanghaftigkeit ist also entweder gar kein Handeln ( $\mathrm{r}$ ) oder eines, über das die Person nur verminderte Kontrolle hat (2), oder die Motive des Handelns, z.B. irrationale Ängste, sind zwanghaft (3). Gehen wir diese Optionen der Reihe nach durch.

Ad I): Ist Zwanghaftigkeit ein unbeabsichtigtes Verhalten?

Es gibt einen merkwürdigen Zusammenhang zwischen nichtkontrolliertem und überkontrolliertem Verhalten. In beiden Fällen ist der Betroffene einem Verhalten ohnmächtig ausgeliefert. Aber dennoch kommen wir nicht auf die Idee, jeden Zornausbruch als zwanghaft zu bezeichnen. Hemmungslosigkeit ist eher eine Auflehnung gegen Zwang als eine Unterwerfung unter ihn. Im Gegensatz zum unkontrollierten Ausdrucksverhalten haben Ticks schon eher etwas mit Zwanghaftigkeit zu tun. Sie entstehen ungewollt, sind dem Betroffenen unangenehm, drängen sich ihm auf. Aber gerade ihr ungewolltes Vorkommen disqualifiziert sie als zwanghafte Symptome, denn Zwang impliziert, daB man etwas unter Druck (aus Angst vor negativen Folgen) wählt. Ticks erscheinen dissoziiert von (bewuBten) psychischen Ursachen und sind deswegen unkontrolliert, aber nicht zwanghaft.

Aber selbst wenn sich herausstellen sollte, daB einige nicht-intentionale Verhaltensweisen als zwanghaft zu qualifizieren sind, würde dadurch höchstens ein unwichtiger Aspekt des Begriffs erläutert. Relevante Phänomene wie das umständliche Befolgen von sinnlosen Regeln, phobisches Vermeidungsverhalten, repetitive Konfliktmuster (Wiederholungszwang), gehören offensichtlich in den Kontext von Handlungen.

\section{Ad 2): Ist Zwanghaftigkeit ein Verlust von Handlungskontrolle?}

Läßt sich eine Form von Handeln denken, das von dem Handelnden nicht kontrollierbar ist? Ist zwanghaftes Handeln identisch mit einem solchen unkontrollierbaren Handeln? Culver und Gert (1982, II3-II7) vertreten diese These. Handeln impliziert für diese Autoren nicht die Fähigkeit, sich zwischen Alternativen zu entscheiden, zumindest kann diese Fähigkeit in ho- 
hem Grade eingeschränkt sein. In solchen Fällen leiden die Personen an einem Verlust von Kontrolle. Ihre Wünsche lösen Handlungen aus, die sie lieber unterlassen würden, oder hindern sie an Handlungen, die sie lieber tun würden. Solche Handlungen sind intentional, aber nicht freiwillig (kontrolliert). Denn freiwillige Handlungen enthalten neben dem Wunsch, sie zu vollziehen, außerdem Kontrollfähigkeit. Mit Bezug auf freiwillige Handlungen kann der Handelnde wählen, ob er sie ausführen oder abbrechen möchte. Er hat die Freiheit, etwas zu tun oder nicht zu tun, je nachdem, ob er will oder nicht. Damit - so Culver und Gert - ein Verhalten als Handlung zu klassifizieren ist, genügt, da $B$ es gewollt wird, und da B die Person fähig zu diesem Verhalten ist. Damit die Handlung freiwillig erfolgt, mu $B$ die Person auch fähig sein, die Handlung effektiv nicht zu wollen, und d.h. fähig sein, die Handlung zu unterlassen, sofern sie will. Zwanghafte Handlungen sollen nun solche sein, die gewollt, aber aus Mangel an Handlungskontrolle nicht freiwillig sind. Culver und Gert entnehmen ihre Beispiele dem Manual für psychische Störungen der amerikanischen Psychiatrie. Als Beispiel für die Unfähigkeit, einen Typus von Handlung zu unterlassen, dient der zwanghafte Händewascher, der in seinem Ritual fortfährt, ohne daB Drohungen oder Belohnungen ihn davon abhalten können. Als Beispiel für die Unfähigkeit, bestimmte Handlungsalternativen zu wählen, dient der Phobiker, der unter allen Umständen vermeidet, Aufzüge zu betreten. SchlieBlich werden noch Personen erwähnt, die Ärzten Symptome vorspiegeln und bereit sind, für unnötige Behandlungen zu leiden oder sich einsperren zu lassen. Diese Festlegung auf eine Patientenrolle wird bei diesen Personen nicht um äuBerer Vorteile willen angestrebt (z.B. um vom Wehrdienst befreit zu werden). ${ }^{\text {I }}$

Culver und Gert glauben, $\mathrm{daB}$ es einen empirischen Test gibt, der die Hypothese vom Verlust der Kontrollfähigkeit zu verifizieren erlaubt. Dieser Test besteht grob gesagt darin, daB man einer Person gute Gründe gibt, sich gegen die mutmaBlich zwanghafte Handlungsalternative zu entscheiden. Gute Gründe, eine Handlung nicht zu wählen, sind etwa die Drohung mit negativen Folgen und/oder die Belohnung von alternativen Handlungen. Culver und Gert unterscheiden zwischen zwingenden und nicht-zwingenden Anreizen, Handlungsalternativen zu wählen. Beispiele für zwingende Anreize wăren das Vermeiden von Schmerz, Körperverletzung, Freiheitsverlust oder Tod. Nicht-zwingende Anreize wären dagegen Belohnungen. Wenn keinerlei Anreize bewirken können, da B die Person ihre Handlungsweise ändert, dann mangelt es dieser an Kontrollfähigkeit und sie ist eben deswegen zwanghaft.

Ein solches Testverfahren stößt auf den Einwand, daß empirisch kaum festzustellen ist, ob eine Person eine Handlungsalternative tatsächlich auf der Basis ihrer Präferenzen und Motive wăhlt oder blo $B$ weil sie unfähig ist, anders handeln zu können. So sind phobisches Vermeidungsverhalten und Zwangsrituale durch Angst vor negativen psychischen Folgen moti- 
viert. Entgegen der These vom Kontrollverlust legen diese Beispiele die Interpretation nahe, daß die betreffenden Personen ihr Verhalten wählen, um nicht in Panik zu geraten. Sie haben irrationale Angst davor, anders zu handeln. Auch bei den Fällen der Simulation von Symptomen glauben Psychiater, daB das Verständnis der individuellen Biographie eine ungewöhnliche Wertschătzung der Patientenrolle offenbart. Es scheint also, daB die Beispiele dafür sprechen, daß Personen die zwanghaften Verhaltensweisen wählen, also nicht so handeln aus Unfähigkeit zu alternativen Handlungen.

In Ermangelung empirischer Beispiele für Handlungen, die Personen aufgrund ihrer Unfăhigkeit ausführen, die präferierte Alternative zu wählen, können sich Culver und Gert nur auf die logische Möglichkeit eines unkontrollierten Handelns zurückziehen. Aber selbst diese logische Möglichkeit ist unausgewiesen, da die Unterscheidung zwischen Fehlleistungen, nichtintentionalem Ausdrucksverhalten und unkontrollierten Handlungen unklar bleibt. Denn nach Culver und Gert handelt eine Person immer dann, wenn sie ein Verhalten zeigt, das sie wünscht und zu dem sie fähig ist. Diese Annahme ist aber unplausibel, denn dann wären unkontrollierte Wutanfälle, verbale Ausrutscher und viele Fehlleistungen Handlungen. Üblicherweise entschuldigt man sich für solche 'Ausrutscher' mit der Bemerkung, da B sie nicht beabsichtigt waren, und insofern liegt es nahe, da $B$ es von Wünschen verursachte Verhaltensweisen gibt, die keine Handlungen sind. Abgesehen davon gilt hier das Gleiche wie bei $\mathrm{r}$ ): es existieren viele unkontrollierte Verhaltensweisen, die keineswegs zwanghaft sind. Die Autoren versäumen es, einen Begriff von Handlung vorzulegen, der deskriptiv zeigt, wie sich Handeln ohne Alternativen denken läBt. Der Standardbegriff von Handeln schlieBt im Gegensatz zu Culver und Gert Kontrollfähigkeit ein.

Aber gibt es nicht doch Beispiele, die nahelegen, Zwanghaftigkeit wenigstens als partiellen Verlust von Kontrollfähigkeit aufzufassen? Frankfurt beschreibt einen Fall von Willensschwäche bei einem Drogensüchtigen, der dies suggeriert:

"Er versucht alles, von dem er meint, daB es ihm ermöglichen wird, den Wunsch nach den Drogen zu überwinden. Aber diese Wünsche sind zu mächtig, um ihnen zu widerstehen und am Ende besiegen sie ihn immer wieder. Er ist ein unwilliger Süchtiger, hilflos vergewaltigt durch seine eigenen Wüsche." (Frankfurt 1971, 12)

Dieser widerwillige Süchtige kann sich nicht handlungswirksam gegen die Drogen entscheiden. Insofern könnte man meinen, da B seine Kontrollfähigkeit eingeschränkt ist und dies wäre der Grund, ihn als zwanghaft zu bezeichnen.

Aber möchte der Süchtige wirklich einer anderen Präferenz als der des Drogenkonsums den Vorzug geben? Gewiß, er wünscht, das Bedürfnis nach 
Drogen wäre nicht so stark, und er kommt sich schlecht vor, weil er es sich erfüllt. Auch stuft er ein Leben ohne Drogenkonsum höher ein als eines mit Rauschgift. Wenn er sich all dessen bewuBt ist und sich dennoch für die Drogen entscheidet, ist dies ein Grund zu sagen, er könne sich in seinem Verhalten nicht gegen die Drogen entscheiden? Dies wäre nur dann plausibel, wenn sinnvoll gedacht werden könnte, daß die Kraft des Drogenwunsches hinreicht, den Versuch zu vereiteln, sich gegen ihn zu entscheiden. Ist es nicht plausibler anzunehmen, da B die Person im Konflikt zwischen Ichidealen und Bedürfnis nach Rauschgift sich gegen jenes entscheidet?

Um einen Fall wie den des Rauschgiftsüchtigen zu beschreiben, bedarf es nicht des Rekurses auf Zwanghaftigkeit, sondern blo $B$ der Unterscheidung zwischen den Gesichtspunkten: "Was möchte ich tun?" und: "Wer möchte ich sein?" Der erste Gesichtspunkt richtet sich auf Weltveränderung, der zweite auf Selbstveränderung. ${ }^{2}$ Der auf Weltveränderung zielende Gesichtspunkt geht von den Bedürfnissen oder Präferenzen aus, die eine Person nun einmal hat. In diesem Sinn könnte der Süchtige zu dem Ergebnis kommen, da $B$ er nicht ohne die Befriedigung seiner Sucht leben kann, obwohl diese Handlungsweise seinen Vorstellungen von wertvollem und gesundem Leben widerspricht. Dieses Sich-schicken in die Sucht kann er durch einen therapeutischen Handlungsvorsatz zur Selbstveränderng ergänzen: "So wie ich bin, kann ich die Qual nicht aushalten, ohne Rauschgift zu leben. Aber mir wäre nichts lieber als frei zu sein von meiner Abhängigkeit und deshalb unternehme ich etwas, um von der Sucht geheilt zu werden." Dieser widerstrebende Süchtige ist negativ zu seiner Sucht eingestellt, leidet in seiner Selbstachtung, wenn er seine Sucht befriedigt, und versucht, seine Bedürfnisse zu verändern. Seine Absicht zielt darauf, das eigene Präferenzsystem zu revidieren.

Der Süchtige tut das, was ihm unter der Voraussetzung seines negativ bewerteten Präferenzsystems am Besten erscheint: er befriedigt seinen unerträglichen Drang, aber er will diesen Drang nicht und versucht sich deshalb zu verändern. Etwas 'wider den eigenen Willen tun' bedeutete dann Präferenzen nachzugeben, die dem Selbstideal der Person widersprechen. Der Süchtige tut etwas, was dem widerspricht, wie er sein möchte, und er tut auBerdem etwas, um in Zukunft seinem Wunschbild entsprechen zu können. 'Wider den eigenen Willen' handelt eine Person, wenn sie im Sinne ihres negativ bewerteten Präferenzsystems handelt und gleichzeitig die Absicht hat, letzteres zu verändern.

Die Diskussion der Vorschläge von Culver, Gert und Frankfurt hat gezeigt, da B sich Zwanghaftigkeit weder durch den Verlust noch durch die Einschränkung von Handlungskontrolle erläutern läßt. Soll man daraus schließen, daB "zwanghaftes Handeln" ein verwirrter Begriff ist? 
Ad 3): Ist "Zwanghaftigkeit" Handeln aufgrund von ir rationalen Ängsten?

Eingangs habe ich zwischen drei Versuchen unterschieden, Zwanghaftigkeit zu verstehen. Die beiden ersten Optionen, die Zwanghaftigkeit als jenseits von Handeln bzw. als unkontrolliertes Handeln deuten, waren ein Fehlschlag. Jedoch bleibt eine dritte Möglichkeit: Zwanghaftigkeit im Sinne von (zwanghafter) Belastung bzw. Hemmung durch irrationale Probleme aufzufassen. Dabei lassen diese Probleme die von ihnen belasteten Personen nicht unbewegt, sondern gehen mit großer Angst einher. Es wird sich zeigen, daß das wichtigste Element von Zwanghaftigkeit irrationale Ängste sind. Man denke an Menschen, die leere Plätze vermeiden, um nicht in Panik zu geraten, oder aus demselben Grund Rituale wie ständiges Händewaschen ausführen. Wenn man annimmt, da $B$ Handlungen dann als von innerem Zwang motiviert zu beschreiben sind, wenn sie dazu dienen, um von irrationalen Ängsten zu befreien bzw. sie zu vermeiden, dann ist zu vermuten, da B das Verständnis von 'irrationaler Angst' die wichtigste Voraussetzung dafür ist, um inneren Zwang und Zwanghaftigkeit zu verstehen.

\section{Theorien irrationaler Angst}

Wie kann man vor etwas Angst haben, das man gleichzeitig für harmlos oder absurd hält? So hält derjenige, der Angst vor Aufzügen hat, die Gefahr, mit Aufzügen zu fahren, für geringfügig; und derjenige, der Angst davor hat, seine Kinder umzubringen, hält dieses Ziel für abwegig. Soll man sagen, da $B$ Angst nichts mit einer Beurteilung dessen zu tun hat, wovor man Angst hat? Ist Angst etwas Ähnliches wie Unlustgefühl, das man hat oder nicht hat, und das gänzlich unabhängig davon ist, ob man etwas als gefährlich einschätzt? Nun gibt es rationale und irrationale Ängste, aber keine rationale oder irrationale Unlust. Eine Theorie, die sagt, Angst sei so etwas Ähnliches wie ein Schmerz, müßte zu der Ansicht kommen, daß im strengen Sinn die Einschätzung der Rationalität der Angst nichts mit dem Gefühl der Angst zu tun hat, sondern die Evaluation der Rationalität sich alleine auf das Urteil bezieht, mit dem eingeschätzt wird, ob die Angst von einer wirklichen Gefahr kündet oder nicht. In einem ähnlichen Sinn könnte man dann auch von einem 'rationalen' oder 'irrationalen' Schmerz sprechen, je nachdem, ob er von einer Krankheit herrührt oder nicht. Jedoch erscheint die Rede vom rationalen bzw. irrationalen Schmerz keineswegs so natürlich wie die von der 'rationalen' bzw. 'irrationalen Angst'. Der Grund dafür ist, daß die Ursache des Schmerzes diesem äußerlich bleibt. Es gibt ein 'Wovor der Angst', aber nichts Entsprechendes beim Schmerz oder der Unlust. Ist dieser dem Begriff "Angst" inhärente Objektbezug beliebig oder ist es nicht doch merkwürdig, wenn eine Person sagt, sie habe vor etwas Angst, das völlig harmlos sei? Dies könnte (entgegen der Theorie, die Angst auf eine Art Unlust reduzieren 
möchte) daran liegen, daß der Begriff "Angst" das Urteil impliziert, daB eine Situation gefährlich ist. Die entsprechende These lautet, daB jemand sich nur dann in vollem Sinne Angst zuschreibt, wenn er glaubt, von etwas bedroht zu sein. Nennen wir das 'die Urteilstheorie der Angst'. Es scheint eine Konsequenz dieser Theorie zu sein, daB irrationale Angst irgendwie unverständlich ist, da in ihr ja kein Urteil enthalten zu sein scheint, daB eine gefährliche Situation besteht. Stimmt die Urteilstheorie und wenn ja, in welchem Sinn ist dann irrationale Angst unverständlich?

\section{I Ist irrationale Angst unverständlich?}

Nehmen wir an, Angst sei nur dann 'im vollen Sinn' gegeben, wenn ihr Träger sich in Gefahr wähnt. Im Widerspruch dazu steht das Phänomen irrationaler Angst, die zwanghaftem Verhalten zugrunde liegt. Wie können harmlos erscheinende Situationen Ängste auslösen? Ich will zuerst die Auffassung untersuchen, daB dieser Widerspruch ein Abweichen von den Standardfällen von Angst bedeute. Irrationale Ängste sind danach gerade deswegen ein pathologisches Phänomen, weil sie nicht auf den für Ängsten angemessenen Urteilen beruhen. So ist der Phobiker seinen eigenen affektiven Fehlurteilen ausgesetzt, wobei er weiB, da B es sich um Fehlurteile handelt. Da in diesen Fällen "Angst" anders verwendet wird als in der durch die Urteilstheorie vorgeschriebenen Weise, wird gefolgert, daB der Phobiker keine 'richtige' Angst habe, da B er sich nicht richtig versteht.

Der Phobiker hat vor harmlosen Situationen Angst, wohingegen der Zwangsneurotiker Angst hat vor den eigenen Strebungen. Man denke an den Fall eines Vaters, der unter der Vorstellung leidet, er könnte seine eigenen Kinder umbringen, und der alle möglichen AbwehrmaBnahmen trifft, um dies zu verhindern. ${ }^{3}$ Weder als Mittel noch an sich erscheint dem Vater der Gedanke an den Kindermord erträglich, geschweige denn wünschenswert. Soweit es ihm bewuBt ist, liebt er seine Kinder und der Gedanke, da B sie getötet werden könnten und gar noch von ihm, ist ihm unfaßbar. In solchen Fällen ist nicht nur die Angst irrational, sondern auch die Annahmen uber die eigenen Ziele. Wie kommt der Vater dazu, sich ein Ziel zuzuschreiben, das er in keiner Hinsicht als wünschenswert begreift? Ist es nicht ein Widerspruch zu sagen, man habe ein Ziel, aber dieses erscheine einem in jeder Hinsicht als schlecht?

Akzeptiert man die These, daB irrationale Ängste, aber auch die mörderischen Gedanken jenes unglücklichen Vaters, ihren Trägern partiell unverständlich sind, so ist daraus zu folgern, da B sich zwanghafte Personen psychische Zustände zuschreiben, die diesen Status nicht in vollem Sinn beanspruchen können. Denn ihre Zwecke und Ängste implizieren Urteile, die sie selber für falsch halten. 
Will man die Störung im Selbstverständnis, die nach dieser Auffassung vorzuliegen scheint, näher spezifizieren, so liegt, wenn man die Urteilstheorie der Gefühle unterstellt, die Auffassung nahe, daB Personen, die sich irrationale Ängste zuschreiben, gegen elementare Verwendungsregeln der Begriffe für das psychische Vokabular verstoßen. Der Fehler betrifft die Funktion bzw. das sog. 'formale Objekt' intentionaler Einstellungen. ${ }^{4}$ Mit 'formalem Objekt' ist gemeint, $\mathrm{da} B$ intentionale Einstellungen sich nicht auf beliebige Gegenstände beziehen können, sondern da $B$ es für diesen $B e-$ zug begriffliche Restriktionen gibt. Auch wenn man nicht weiB, worauf sich eine Meinung bezieht, steht begrifflich fest, daB eine Person nur das meinen kann, was sie für wahr hält. Das formale Objekt von Meinungen ist ihre Wahrheit, das von Wünschen das Gute und das von Gefühlen das Bedeutsame. Meinungen dienen dazu, auszudrücken, was Personen für wahr halten; Wünsche beschreiben Sachverhalte, deren Existenz Personen mindestens in einer Hinsicht für wünschenswert halten; und Gefühle heben an Situationen hervor, was Personen bedeutsam erscheint. Meinungen, die Personen nicht als wahr vorkommen, sind nicht ihre Meinungen; Wünsche, deren Erfüllung man in keiner Hinsicht gut findet, sind nicht die eigenen Wünsche; und zu Situationen, die einen nicht berühren, ist man nicht emotional eingestellt. Nach dem bisher Gesagten hat es also den Anschein, daB zwanghafte Einstellungen solche sind, die 'sich aufdrängen', obwohl sie den genannten Ansprüchen nicht genügen. Es sind Einstellungen, denen in dem oben genannten Sinn das formale Objekt fehlt. Zwanghafte Handlungen wären dann solche, diẹ durch solche defekten Einstellungen motiviert sind.

Der Phobiker versteht nicht, warum ihn harmlose Objekte so stören. Der unter Waschzwang Leidende versteht nicht, wieso es (für ihn) erstrebenswert ist, sich so oft zu waschen. Die Auseinandersetzung mit unverständlichen Aspekten der eigenen Person hindert sie in diesen Fällen das zu tun, was ihr am besten scheint. Denn eine notwendige Voraussetzung dafür, da $B$ Personen frei entscheiden können, was sie tun möchten, besteht darin, da $B$ ihnen die Entscheidungsalternativen verständlich sind. Wenn eine Person Urteilen 'ausgeliefert' ist, die sie nicht für wahr, oder Zwecken, die sie nicht für erstrebenswert hält, läßt sich sagen, daß ihre Fähigkeit, das zu tun, was sie möchte, auf der Basis von psychischen Ursachen gestört ist. Zwanghaftigkeit schränkt die Selbstbestimmung ein, da die Betroffenen durch ihnen unverständliche psychische Faktoren gehindert werden, das zu tun, was ihnen am besten erscheint.

Der Gang der Untersuchung bisher läßt sich folgendermaßen zusammenfassen: Die Meinung, da $B$ Zwanghaftigkeit in einem (graduellen) Verlust von Handlungskontrolle besteht, wurde zurückgewiesen. Zwanghaft ist nicht das Verhalten, sondern sind die Einstellungen des Zwanghaften, die sich durch Einsichten des Betroffenen nicht verändern lassen. Das Charakteristische von zwanghaftem Handeln ist seine Motivierung durch irrationale 
Ängste. Ihre Irrationalität besteht in ihrer partiellen Unverständlichkeit. Der Zwanghafte hat Angst vor Sachverhalten, die er gleichzeitig als harmlos beurteilt, oder er hat Angst, daB er etwas tun könnte, was ihm verbrecherisch und absurd erscheint. Während Drohungen im Fall des äußeren Zwangs ihren Ursprung in anderen Personen haben, gehen sie beim inneren Zwang von der Person selber aus. Der Zwanghafte stößt beim inneren Zwang auf Verständnisgrenzen: Er fühlt sich gezwungen, sich Ziele zuzuschreiben, deren Erfüllung er nicht wünscht, auf Urteile zu hören, die er für falsch hält. Man kann die zuletzt genannte Erläuterung von Zwanghaftigkeit als die 'Unverständlichkeitsthese' bezeichnen.

\subsection{Die Unverständlichkeit der Unverständlichkeitsthese}

Die Unverständlichkeitsthese ist dem naheliegenden Einwand ausgesetzt, selber unverständlich zu sein. Denn wenn im Falle von Zwanghaftigkeit semantische Sinnbedingungen von intentionalen Einstellungen verletzt werden, dann taucht die Frage auf, wie derjenige, der diese sogenannten Einstellungen hat, diese uberhaupt identifizieren kann. Was soll es heißen, da $B$ dem Zwanghaften seine eigenen Einstellungen unverständlich sind?

Ich sehe drei Möglichkeiten, diese Unverständlichkeit zu verstehen:

I) Die irrationale Angst des Zwanghaften ist gar keine richtige Angst, sondern ein intensives Unlusterleben.

2) Zwanghafte haben ein unvollständiges Wissen von ihrer Angst. Sie identifizieren zwar ihre Angst richtig, aber machen einen Fehler bei der Identifikation des Gegenstands der Angst.

3) Irrationale Ängste und Absichten sind den Betroffenen nicht semantisch, sondern narrativ und kausal unverständlich. Der Zwanghafte versteht nicht, warum er jene Ängste und Ziele hat.

Gehen wir die Möglichkeiten der Reihe nach durch.

Ad I): Es ist bei Zwanghaften zwischen zwei Ängsten zu unterscheiden: Dem Angstanfall und der Angst vor dem Angstanfall. Da ist erstens die Angst davor, in die gefürchteten Situationen zu gelangen. Diese Angst speist sich aus dem Wissen um die Disposition, unter bestimmten Bedingungen Angstanfälle zu entwickeln. Zweitens die Angstanfälle in den gefürchteten Situationen. Beide Ängste sind für das Verständnis des Phänomens der Zwanghaftigkeit wichtig. Die Angst vor dem Angstanfall zwingt Personen, bestimmte Situationen zu vermeiden. Damit wird ihr Handlungsspielraum eingeschränkt. Der extreme Typus von Fällen ist der, in dem eine Person bestimmte Bedingungen herstellen muB, um Angstanfälle zu vermei- 
den, z.B. Händewaschen, spitze Gegenstände wegräumen, sinnlose Suchrituale ausführen. An solchen Beispielen erkennt man den begrifflichen $\mathrm{Zu}$ sammenhang zwischen Phobien und Zwangssymptomen. Das Auftreten von irrationalen Angstanfăllen unter voraussehbaren Bedingungen ist beiden gemeinsam. Nur sind die Auslöser der Angstzustände im Fall der Phobie wahrgenommene Zustände der Welt und im Fall der Zwangsneurose psychische Zustände.

Die Angst vor dem Angstanfall ist rational. Hier hat die Person Angst davor, schreckliche Erfahrungen zu machen. Dies gehört zu den Standardfällen von Angst und stellt insofern kein konzeptuelles Problem dar. Ein solches Problem entsteht erst durch den Angstanfall selbst. Könnte man aber dem Problem nicht entgehen, indem man Phobikern und Zwangsneurotikern statt Angstanfällen ungewöhnliche, intensive Unlusterlebnisse zuschreibt? Diese Unlust wäre ungewöhnlich, da sie weder durch Zufügung von Schmerz noch durch das BewuBtsein von Gefahr ausgelöst wird. Statt zu sagen, der Phobiker habe Angst vor irrationalen Angstanfällen, könnte man sagen, er habe Angst vor ungewöhnlichen Unlusterlebnissen.

Eine solche Beschreibung scheitert aber grundsätzlich bereits an ihrer phänomenalen Unangemessenheit, da die Zwanghaften in der Tat Angst vor leeren Plätzen erleben oder sich manchmal tatsächlich zutrauen, da B sie ihre Kinder umbringen könnten.

Ad 2): Gehen wir daher zu der zweiten Möglichkeit über, daB irrationale Angst in einem unvollständigen Wissen besteht. Die Person identifiziert die eigene Angst richtig, aber ihr Objekt falsch. Dies würde zu der Auffassung von Freud passen, der behauptet, daB die phobischen Ängste auf der Basis von Verschiebung gebildet werden. An die Stelle des unbewuBten Objekts der Angst schiebt sich ein bewuBter Stellvertreter. So wie eine Bemerkung einen Ärger auslösen kann, der nicht ein Ärger über jene Bemerkung ist, so müBte man auch bei der Phobie zwischen dem unterscheiden, wovor die Person tatsächlich Angst hat, und dem vordergründigen Auslöser der Angst. Dies setzt voraus, da B eine Person den Zustand der Angst identifizieren kann, ohne $d a B$ ihr bewuBt ist, wovor sie Angst hat. Nach dieser Auffassung wäre eine irrationale Angst eine zum Teil unbewuBte Angst. Unbewußt wäre das Objekt, vor dem sich der Phobiker fürchtet.

Nun ist aber fraglich, ob die Unterscheidung zwischen dem Auslöser und dem Gegenstand von Gefühlen bei der Beschreibung von Phobien und Zwangsneurosen weiterhilft, denn hier haben die Betroffenen klare Vorstellungen daruber, wovor sie Angst haben. Soll man sagen, der Phobiker irre sich darin, daB er z.B. Angst vor leeren Plätzen hat? Zwar spricht viel dafür, da $B$ Zwanghafte nur ein sehr unvollständiges Wissen über die motivationalen Bedingungen ihrer Störung haben, aber dies ändert nichts 
daran, da B sie sehr genau wissen, vor was sie Angst haben. Dies scheint der dritten These von der narrativen Unverständlichkeit der irrationalen Ängste Recht zu geben.

Ad 3): Der Phobiker versteht nicht, warum harmlose Umstände solche Unlust bei ihm hervorrufen, und der an Zwangssymptomen Leidende kann den Zusammenhang zwischen Unlustvermeidung und ihm ansonsten absurd anmutenden Ritualen nur registrieren, aber nicht verstehen. Insofern scheint es zutreffend, daB die irrationalen Zustände den Betroffenen narrativ und motivational unverständlich sind. Zwanghafte könnten dann irrationale Episoden zwar semantisch verstehen, aber biographisch nicht integrieren. Eine Möglichkeit, narrative Unverständlichkeit mit semantischer Verständlichkeit kompatibel zu machen, bestünde in der Vorstellung, daß Zwanghafte die Einstellungen, die sich ihnen aufdrängen, nach dem Muster des Fremdverstehens begreifen. Denn wir können Einstellungen (Wünsche, Urteile, Gefühle) von anderen verstehen, obwohl wir sie nicht teilen.

Doch dagegen spricht, daB jene narrativen Fremdkörper Angst bereiten. Denn wären die sich aufdrängenden Einstellungen blo $B$ fremd, dann ist nicht einzusehen, warum sie die Betroffenen ängstigen sollen. Mit dem Modell des Fremdverstehens läßt sich erläutern, da $B$ Zwangsgedanken stören, aber nicht, daB sie Panik auslösen. Es ist deshalb plausibler, die narrativ desintegrierten Inhalte mit einem Modell von Ichspaltung zu erfassen. Damit ist gemeint, daB die Person sich in ihren Angstanfällen kognitiv und motivational verändert. Der Phobiker würde demnach während des Angstanfalls wirklich glauben, daB leere Plätze gefährlich sind, jener unglückliche Vater würde sich episodisch tatsächlich zutrauen, seine Kinder umzubringen usf. Die narrativ unverständlichen Episoden bezögen ihre motivationale und affektive $\mathrm{Kraft}$ somit aus irrationalen Urteilsveränderungen. 'Irrational' hat hier genau den Sinn, den Gert in diesem Zusammenhang erläutert: Es sind falsche Überzeugungen, gegen die überwältigend viel spricht und die mit dem übrigen Wissen der Person unvereinbar sind ( $s$. Gert, in diesem Heft).

Beck (1979, Kap. 7) hat am Beispiel der Phobien versucht, diese These von der irrationalen Urteilsveränderung zu untermauern. Er hat herausgefunden, daß Phobiker die Objekte ihrer Ängste nur so lange als harmlos beurteilen, als sie nicht unmittelbar mit ihnen konfrontiert werden. Die Einschätzung der Gefährlichkeit der Inhalte der Phobien vergrößert sich proportional zur Nähe zu den phobischen Situationen. Beck hat diese These empirisch untersucht. Er schreibt:

"Bei vielen Anlässen habe ich die Patienten in die phobische Situation begleitet (ich bin mit ihnen beispielsweise Treppen gestiegen, ins Wasser gegangen oder mit einem Fahrstuhl gefahren), und ich konnte dabei ihre zunehmende Erwartung eines Unheils verifizieren .... Als ich eine Frau, die an Höhenangst litt, auf einer Bergwanderung begleitete, wurde ihr plötz- 
lich schwindlig, sie begann zu schwanken und 'fühlte' eine Kraft, die sie in die Tiefe zog. Im 40. Stockwerk eines Wolkenkratzers 'fühlte' sie, wie sich der FuBboden steil nach unten zu neigen begann. Eine Frau, die sich vor dem Wasser fürchtete, sah sich selbst ertrinken, obwohl sie sich blo $B$ am Strand aufhielt. Sie begann nach Luft zu ringen, als ob sie tatsächlich ertrinke." (Beck 1979, 139)

Folgerichtig definiert Beck Phobien als "Furcht vor einer Situation, die nach allgemeinem Konsens und dem eigenen Urteil des Betreffenden, wenn dieser der Situation fern ist, in keinem Verhältnis a) zu der Gefährlichkeit der speziellen Situation und b) der Wahrscheinlichkeit der Gefährdung in dieser steht" (134).

Die irrationalen Angstanfälle werden für Beck vermittelt durch irrationale Urteilsveränderungen. Diese Auffassung erinnert an die Theorie der Willensschwäche von Aristoteles: Wünsche verursachen, daB Willensschwache den klaren Kopf verlieren. Sie vergessen, was für sie am besten ist und befriedigen ihre unmittelbaren Wünsche. Genauso verlieren Phobiker in bestimmten, sogar von ihnen voraussehbaren Situationen ihre Fähigkeit zu überlegen und fällen irrationale Urteile, die Ängste verursachen. Auch die Zwangssymptomatik läßt sich durch Verlust der Fähigkeit zu überlegen erläutern. Während des Zwangsanfalls glaubt der Betroffene, daB er z.B. seine Kinder umbringen könnte, und da $\mathrm{B}$ er dies nur durch bizarre Vorsichtsmaßnahmen vermeiden kann. In dieser Situation ist er nicht fähig, die Absurdität des Gedankens und seiner Abwehrmaßnahmen einzusehen. Die Unfähigkeit, sich von Gedanken zu distanzieren, sie einzuschätzen und abzulehnen, scheint deshalb immer eine notwendige Bedingung von Zwanghaftigkeit zu sein.

Um zusammenzufassen: Es war nicht klar, wie irrationale Ängste zu verstehen sind. Die Urteilstheorie der Gefühle schien hier an eine Grenze zu stoßen. Es hat sich aber gezeigt, daß vielen Phänomenen irrationaler Angst irrationale Urteilsveränderungen zugrundeliegen. Charakteristisch für zwanghaftes Handeln ist der Versuch, Angstanfälle zu vermeiden. Ihr Auftreten ist vermittelt durch die Bildung irrationaler Überzeugungen, die verbunden sind mit der Blockierung der Fähigkeit zu überlegen. Man kann dies die 'partielle Unzurechnungsfähigkeitsthese' nennen.

\subsection{Irrationale Angst als Dissoziation von Erfahrung und Urteil}

Ich denke, daß viele, aber nicht alle Phänomene irrationaler Angst durch irrationale Urteilsveränderung zu verstehen sind. Daneben gibt es Fälle, in denen die Betroffenen Angstanfälle erleiden und gleichzeitig wissen, da B die Situationen harmlos sind. Selbst einige Beispiele von Beck sind auf diese Weise zu beschreiben. So nennt Beck eine Bergsteigerin, die nicht schwindelfrei ist und auf Bergen fühlt, daB eine Kraft sie nach unten 
zieht, oder die im 40. Stock eines Wolkenkratzers 'sieht', wie der Boden sich senkt. Es stellt sich die Frage, ob solche verunsichernden Sinnestäuschungen Meinungen implizieren. MuB derjenige, der in Panik gerăt, weil er fühlt, daB eine Kraft ihn in den Abgrund zieht, glauben, da B so eine Kraft existiert? Ist es begrifflich notwendig, daB einer verzerrten Sichtweise unangemesssene Meinungen korrespondieren? Bei Sinnestäuschungen ist das Phänomen bekannt, $\mathrm{da} B$ eine Person 'sieht', da B ein Stab im Wasser gebrochen erscheint, obwohl sie keineswegs an diesen Schein glaubt.

Man könnte nun meinen, daB im Falle von Angst das entsprechende evaluative Urteil deswegen notwendig sei, weil ohne ein solches Urteil das Erleben einer ernsthaften Bedrohung wegfalle. Wenn die Frau weiB, daB der Boden sich in Wirklichkeit nicht senkt, dann wird sie keine Angst mehr davor haben, in die Tiefe zu rutschen. Aber dies ist keineswegs zwingend so. Denn das Wissen, daB die Welt nicht so ist, wie man sie sieht, verhilft einem zu keinem sicheren Stand. Genausowenig hilft die Einsicht, daB niemand einen in den Abgrund zieht, gegen das Gefühl, gezogen zu werden. Was nützt es, wenn man weiß, da $B$ man 'nur' unter der Sogwirkung der eigenen Phantasien leidet? Die Kritik der Sinnestäuschungen vermittelt nicht 'neue Sinne'. Phantasien und Pseudowahrnehmungen können Bestandteile von Ängsten sein, denen keine evaluativen und kognitiven Urteile entsprechen. Es ist wichtig, zwischen den Ebenen der Wahrnehmungen, der Phantasien und ihren Interpretationen zu unterscheiden. Wenn dies stimmt, sind nicht immer die Überzeugungen des Phobikers für die Phobie wesentlich, sondern die Phobien können auch auf seinen unmittelbaren Erfahrungen beruhen. Es gibt Fälle, in denen Zwanghafte an Angst leiden, obwohl sie einsehen, daB keine Gefahr droht. In solchen Fällen ist nicht die Fähigkeit zur Argumentation oder Einsicht blockiert, sondern die emotionalen Reaktionen sind argumentativer Beeinflussung unzugänglich. Das Problem des Zwanghaften in diesen Fällen besteht darin, da B seine Sinne $\mathrm{ihm}$ in bestimmten Situationen Streiche spielen. Es kommt zu emotionalen Sinnestäuschungen.

Nun wäre es sicher übertrieben zu behaupten, da B beim Zwanghaften immer Urteil und Gefuhl dissoziiert sind. Es kam mir nur darauf an zu zeigen, daB dieser Fall denkbar ist und vermutlich auch nicht ganz selten vorkommt. Shapiro (1981, 96-100) hat die interessante Beobachtung gemacht, daB Hypochonder sich nie einbilden, eine Krankheit zu haben, sondern immer im Konjunktiv argumentieren. Sie wollen ausschließen, da $B$ sie eine bestimmte Krankheit haben könnten. Bei dem Zwanghaften ist es ähnlich: Er hat die Idee, es könnte passieren, daß er seine Kinder umbringt und hat eine Menge Phantasien, die dies antizipieren. Es wäre falsch zu sagen, er glaube, da $B$ er seine Kinder umbringen wird, wenn er nicht etwas dagegen tut. Aber er schließt diese Möglichkeit nicht völlig aus. Auch wenn das Schreckliche unwahrscheinlich ist, lohnt es sich etwas zu seiner 
Verhinderung zu tun. Man könnte diese Fälle als Übergangsphänomene zwischen Glauben und Nichtglauben bezeichnen.

Zusammenfassend läßt sich sagen: Handlungen werden zwanghaft genannt, wenn sie Versuche sind, irrationale Angstanfälle zu vermeiden. Diese irrationalen Angstanfälle wurden auf zwei Weisen erläutert. Im Rahmen der Urteilstheorie der Gefühle wurde erstens eine irrationale Urteilsveränderung angenommen. Bestimmte Bedingungen rufen regelmäBig Fehleinschätzungen von Gefahren hervor. Die Personen leiden unter Bedrohungen, wenn sie sich in bestimmte Situationen begeben, obwohl sie diese Bedrohungen aus der Distanz als harmlos durchschauen. BewuBte Zwanghaftigkeit setzt das Wissen voraus, daß bestimmte Umstände solche irrationale Einschätzungen auslösen. Zwangsrituale sind Mittel, um die Entstehung solcher irrationalen Fehlurteile zu verhindern. Zweitens hat sich gezeigt, daB es Fälle gibt, in denen es zu keiner Urteilsveränderung, sondern zu einer Dissoziation zwischen Erfahrung und Urteil kommt. Personen machen entsetzliche Erfahrungen, ohne diese Erfahrungen zur Grundlage ihrer Situationseinschătzung zu machen. So fühlen sie etwa, daB eine Kraft sie in die Tiefe zieht und wissen gleichzeitig, daB dieses Gefühl blo $B$ ein Produkt ihrer Einbildungskraft ist. Der Träger der Angst wei $B$, da $B$ die Bedrohung in Wirklichkeit Produkt seiner eigenen Subjektivität ist. Er weiß, daß seine Erfahrungen ihm keine Einsicht in seine Situation liefern. Bedrohlich ist für ihn nicht eine bestimmte objektive Situation, sondern die Qualität seiner Erfahrungen.

Irrationale Ängste sind daher in manchen Fällen durch irrationale Urteilsveränderung und in anderen durch emotionale Sinnestäuschungen zu erklären.

\section{Ist 'zwanghaftes Handeln' zwanghaft?}

BewuBte Zwanghaftigkeit wurde charakterisiert als ein Verhalten, das dazu dient, irrationale Angstanfälle zu vermeiden. Es sind Handlungen bzw. Handlungseinschränkungen, die aus Angst vor irrationalen Angstanfällen vorgenommen werden. Mit welchem Recht reden wir unter dieser Voraussetzung aber von zwanghaftem Handeln? Wăren dann nicht genaugenommen nur die irrationalen Einstellungen zwanghaft?

Die sogenannten 'zwanghaften' Handlungen entsprechen den üblichen Rationalitätskriterien: Zweckrationalität, Abwendung von Leid und instrumenteller Erfolg. Sie sind zweckrational, denn sie werden ausgeführt, um Panik zu vermeiden. Wenn man wie Gert die Vermeidung von Leid, von dem man selbst bedroht ist, als rational bezeichnet, dann sind zwanghafte Handlungen auch in diesem Sinn rational. SchlieBlich sind sie in vielen Fällen auch erfolgreich: durch Waschrituale oder die Vermeidung der Konfronta- 
tion mit phobischen Objekten werden Angstanfälle vermieden. 5 Kurzum: sogenannte Zwangshandlungen sind (in vielen Fällen) adäquate Mittel zu Zwecken. Sie sollen der Vermeidung von Leid dienen und das ist prima facie ein guter Grund für Handlungen.

Wenn man unter 'zwanghaftem Handeln' ein Handeln versteht, das man zu vollziehen hat, ob man will oder nicht, dann gibt es kein zwanghaftes Handeln. Auch beim Handeln unter äuBerem Zwang wird ja nicht die Fähigkeit, teleologisch zu handeln, eingeschränkt, sondern äußerer Zwang beeinflußt Präferenzen und beschneidet die Menge der Handlungsalternativen. Es ist sinnvoll, sich inneren Zwang in Analogie zu äuBerem Zwang vorzustellen. Außerer Zwang besteht in der intentional bezweckten Einengung des Handlungsspielraums von anderen. Dabei kann der äuBere Zwang entweder das Ziel haben, bestimmte Handlungsmöglichkeiten zu verhindern oder positiv bestimmte Handlungen zu veranlassen. ÄuBerer Zwang bedient sich im großen und ganzen zweier Mittel. Er macht bestimmte Handlungsmöglichkeiten durch Freiheitsberaubung unmöglich (Beispiel: Gefängnisse) oder demotiviert, indem er durch Strafandrohung bestimmte Handlungsmöglichkeiten unattraktiv erscheinen läßt.

Innerer Zwang 'zielt' analog entweder auf die Verhinderung - Beispiel sind die Phobien - oder auf das Bewirken bestimmter Handlungen, z. B. Waschzwang. Ebenso gibt es hier den Unterschied zwischen Handlungen, zu denen Personen aufgrund von psychischen Ursachen nicht in der Lage sind (z.B. irrationale Schüchternheit, psychisch verursachte Arbeitsunfähigkeit) und Handlungen, die Personen deswegen nicht vollziehen, weil sie ihre Folgen fürchten: z.B. extreme Unruhe oder Angstanfälle in bestimmten Situationen.

Wenn eine Person durch äußeren Zwang zu bestimmten Handlungen motiviert wird, so wird häufig gesagt, da $B$ sie in Wirklichkeit nicht ihre eigenen Zwecke verfolge. Damit meint man, daß sie eine bestimmte Möglichkeit nicht wählen würde, wenn nicht ein anderer Drohungen geltend gemacht hätte. Fielen jene Drohungen weg, dann würde sie anders handeln. In einem analogen Sinn sind die Zwecke des Zwanghaften nicht seine Zwecke: Man denke nochmals an das Beispiel des Vaters, der unter der Vorstellung leidet, er könnte seine Kinder töten, und sich veranlaßt sieht, etwas zu unternehmen, um dies zu verhindern. Ohne den Glauben, daß eine ernsthafte Wahrscheinlichkeit existiert, wonach dieser für ihn entsetzliche Fall eintreten könnte, würde er sich nicht zu obskuren Vermeidungsritualen entschlieBen.

Unter innerem Zwang müssen sich Personen mit Hindernissen auseinandersetzen, die denen analog sind, die durch äuBeren Zwang hergestellt werden. Nur existieren im Fall des inneren Zwangs keine externen Instanzen, die Druck ausüben. Insofern ist innerer Zwang irrational. Irrationale Äng- 
ste kann man als innere Sanktionen auffassen. Wer sich ihnen fügt, beugt sich einem inneren Zwang. Wenn man behauptet, da B zwanghafte Handlungen solche sind, die durch irrationale Ängste motiviert werden, so impliziert diese Behauptung also genaugenommen, da $B$ nur die Ängste und die in ihnen enthaltenen Vorstellungen, die sich regelmäßig und wider bessere Einsicht einstellen, zwanghaft sind.

Die These, daB 'Zwanghaftigkeit' nicht die Handlungsfähigkeit, sondern Einstellungen betrifft, die von rationalen Uberlegungen nicht zu beeinflussen sind, bezieht sich nur auf den Begriff 'zweckrationalen Handelns'. In diesem Rahmen hat, wie wir gesehen haben, der Zwanghafte gute Gründe für sein Handeln. Wenn man dagegen untersucht, ob es nicht einen komplexeren Handlungstypus gibt, für den Einstellungen nicht nur als Randbedingung von Handeln relevant sind, sondern der auch die Bildung von Einstellungen mit erfassen möchte, dann würde man vielleich $\mathrm{zu}$ anderen Ergebnissen kommen. Würde es z.B. stimmen, daB Personen ihre Einstellungen auf der Basis eines geteilten Hintergrundwissens und impliziter Rationalitätsannahmen erwerben, so wäre zu fragen, ob nicht Zwanghaftigkeit solche Bildungsprozesse stört. Sollte es sich, wie z.B. Habermas annimmt, als sinnvoll erweisen, diese Bildungsprozesse als Mischung von Kommunikation und Selbstgespräch zu charakterisieren, so würde sich die Frage stellen, ob Zwanghaftigkeit eine solche 'kommunikative Kompetenz' beschränkt. Dies ist wahrscheinlich, da zwanghafte Einstellungen unter normalen Umständen nicht von Überlegungen zu beeinflussen sind und sich daher als Sackgassen für (kommunikative) Bildungsprozesse erweisen. Die Frage, ob der Begriff zweckrationalen Handelns ausreicht, um Sozialisationsprozesse zu erklären, oder ob ein komplexeres Handlungsmodell angemessen wäre, muß hier offen bleiben. Ich nehme aber an, daB der zweckrationale Handlungsbegriff auch im Rahmen von komplexeren Modellen wichtig bleibt.

\section{Zusammenfassung}

Unterschiedliche Ansätze zu einem Verständnis 'bewußter Zwanghaftigkeit' wurden diskutiert. Zuerst wurde die Möglichkeit in Betracht gezogen, da B Zwanghaftigkeit sich nicht in Handlungen, sondern in unbeabsichtigten Verhaltensformen wie Ticks manifestiert. Dabei wurde klar, daB diese Verhaltensphänomene wenig mit innerem Zwang zu tun haben. Als zweite Möglichkeit wurde erwogen, Zwanghaftigkeit mit einem (graduellen) Verlust der Fähigkeit zu erklären, die präferierte Handlungsweise zu wählen. Diese Interpretation lieB sich an Beispielen jedoch nicht erhärten. Stattdessen zeigte sich, da B irrationale Ängste Zwanghafte zu ihrem Handeln motivieren. Dadurch wurde die These nahegelegt, daß Zwanghaftigkeit ein Handeln ist, das dazu dient, irrationale Angst zu vermeiden oder zu beseitigen. Als Folgeproblem stellte sich die Frage, wie irrationale Angst zu be- 
greifen ist. Drei Ansätze wurden untersucht. Die Unverständlichkeitsthese behauptete, daB irrationale Angst eine abnorme Einstellung ist, weil der Begriff "irrationale Angst" den Verwendungsregeln des Begriffs "Angst" widerspricht. Eine Angst vor einer Situation, die gleichzeitig als harmlos beurteilt wird, gilt nach der Unverständlichkeitsthese als sinnwidrig.

Gegen die These von der Unverständlichkeit irrationaler Ängste wurde eingewandt, da B diese Ängste den Betroffenen klar verständlich sind. Deshalb wurden zwei andere Theorien irrationaler Angst vorgeschlagen. Die erste Theorie verweist auf Fälle irrationaler Urteilsverănderung von Personen. Hăufig verändert sich z.B. das Urteil des Phobikers, wenn er sich dem 'phobischen' Objekt nähert. Was er vorher, uberlegend, als harmlos beurteilt hat, beurteilt er nun als lebensgefährlich, wobei er während des Panikanfalls Gegenargumenten nicht zugänglich ist. Diese These, die irrationale Angst durch partielle Unzurechnungsfähigkeit erklärt, eignet sich jedoch nicht zur Erläuterung aller Fälle. Denn es kommt vor, daB Personen, während sie panische Erfahrungen machen, wissen, daß diese nicht zur Grundlage eines Urteils uber ihre Situation taugen. Sie wissen, daB ihre emotionalen Erfahrungen auf Sinnestäuschungen beruhen, was allerdings den unangenehmen Charakter dieser Erfahrungen nicht auflöst. Viele Erscheinungen irrationaler Angst müssen deshalb durch die Annahme einer Dissoziation von Erfahrung und Urteil erklärt werden.

Der Ertrag der vorangegangenen Überlegungen besteht in folgendem. Das Handeln, das als bewuBt zwanghaft klassifiziert wird, ist zweckrational. Es dient der Vermeidung bzw. Auflösung von irrationalen Ängsten. Diese beruhen entweder auf voraussehbar falschen Urteilsveränderungen oder auf einer Dissoziation von Erfahrungen und Urteilen. Die psychischen Erlebnisse, die durch Zwangshandlungen vermieden werden sollen, zeichnen sich durch narrative Unverständlichkeit aus. Aber diese Unverständlichkeit läßt sich nicht einfach im Sinn von Episoden begreifen, die verstanden werden, wie die Erzählungen eines Fremden. Im Gegensatz zu solchem Fremdverstehen beunruhigen die Zwangsgedanken zutiefst. Die Betroffenen glauben während der Angstanfälle an die Richtigkeit ihrer Gedanken oder aber sie werden von emotionalen Sinnestäuschungen geăngstigt.

\section{Anmerkungen}

I In der psychiatrischen Diagnostik heißt dieses klinische Bild 'Münchhausen-Syndrom'. Für eine Fallbescheibung siehe Spitzer et al. 1989, 62 , 99, 150.

2 Die Unterscheidung zwischen Selbst- und Weltveränderung hat Ähnlichkeit mit Pears' Distinktion zwischen 'äuBerer' und 'innerer Intention'. Die außere Intention ist ein Plan, eine (innere) Intention in einer vorausgesehenen Situation auszubilden. Pears nennt das Beispiel von einer Person, die sich vornimmt, nach zwei Drinks aufzuhören und dann ein 
drittes Glas Alkohol trinkt. Aber während äuBere und innere Intention aufeinanderfolgen, können Intentionen, die auf Weltveränderung bzw. auf Selbstveränderung zielen, koexistieren. Im Fall des Süchtigen sind beide Intentionen insofern gegensätzlich, als ihre gleichzeitige Erfüllung ausgeschlossen ist. S. Pears 1984, Kap. ro.

3 Diese Beispiel wird später noch ausführlicher behandelt. Es ist entnommen aus: Thomä/Kächele I988, $528 \mathrm{ff}$.

4 Auf der Ebene von Sprechakten korrespondieren dem formalen Objekt Habermas' 'Geltungsansprüiche'.

5 Hier ist den einflußreichen diagnostischen Kriterien der amerikanischen Psychiater zu widersprechen. Dort wird zwanghaftes Verhalten als Tätigkeit beschrieben, die "nicht in realistischer Weise mit dem verbunden" ist, "was herbeigeführt oder verhütet werden soll ..." DSMIII, 246. Wenn man als Ziel der Zwangshandlungen die Verhinderung von Panikattacken betrachtet, dann sind sie in vielen Fällen 'realistisch' im Sinne von zweckrational.

\section{Bibliographie}

Beck, A.T. (1979), Wahrnehmung der Wirklichkeit und Neurose, München

Culver, C.M./B. Gert (1982), Philosophy in Medicine, Oxford

Frankfurt, H. (197I), Freedom of the Will and the Concept of a Person, in: Journal of Philosophy 68, 5-20

Köhler, K./H. SaB (Hrsg.) (r984), Diagnostisches und statistisches Manual psychischer Störungen (DSM-III, deutsche Ausgabe), BaselWeinheim (zit. DSM-III)

Pears, D. (1984), Motivated Irrationality, Oxford

Shapiro, D. (1981), Autonomy and Character, New York

Spitzer, R.M./M. Gibbon/A.E. Skodol/J. Williams/M. First (eds.) (r989), DSM-III-R Case Book, Washington

Strawson, P.F. (1978), Freiheit und Übelnehmen, in: U. Pothast (Hrsg.), Freies Handeln und Determinismus, Frankfurt, 20r-233

Thomä, H./H. Kächele (1988), Lehrbuch der psychoanalytischen Therapie, Berlin

Tugendhat, E. (1979), SelbstbewuBtsein und Selbstbestimmung, Frankfurt

- $(1984)$, Antike und moderne Ethik, in: E. Tugendhat, Probleme der Ethik, Stuttgart, 33-56 